\title{
Chiral phase diagram in soft-wall AdS/QCD
}

\author{
Sean P. Bartz* \\ Macalester College \\ E-mail: sbartz@macalester.edu \\ Theodore Jacobson \\ Macalester College \\ E-mail: tjacobsiemacalester.edu
}

\begin{abstract}
The holographic connection between strongly-coupled gauge theories and extra-dimensional gravitational theories has succeeded in describing many features of non-perturbative QCD. Phenomenological models called AdS/QCD use a five-dimensional AdS black hole to study thermodynamic properties of the quark-gluon plasma. In this talk, we focus on the phase transition that occurs as chiral symmetry is restored at high temperature and chemical potential. Using a Reissner-Nordström metric for a charged black hole, we find a critical temperature around 150 $\mathrm{MeV}$ and a critical quark chemical potential around $560 \mathrm{MeV}$. We qualitatively reproduce the Columbia plot, showing the dependence of the phase transition order on the light and strange quark masses. We show that the introduction of a chemical potential does not affect the order of the phase transition, implying the absence of a critical point in this simple model.
\end{abstract}

Critical Point and Onset of Deconfinement

7-11 August, 2017

The Wang Center, Stony Brook University, Stony Brook, NY

${ }^{*}$ Speaker. 


\section{Introduction}

The investigation of the phase diagram for quark matter is a major project of nuclear physics. The heavy-ion community is interested in mapping out the phase transition between hadronic matter and the quark-gluon plasma as a function of temperature and baryon chemical potential. In particular, the search for the expected critical point in this phase boundary is the focus of the next run at the Relativistic Heavy Ion Collider (RHIC) at Brookhaven National Laboratory. A robust theoretical description of the phase boundary is thus a worthy goal.

Results from lattice QCD have demonstrated that the deconfinement phase transition at zero quark chemical potential $(\mu)$ is a rapid crossover. Extending lattice results to finite chemical potential is prevented by a well-known obstacle known as the sign problem. Techniques exist for extending lattice QCD to finite but relatively small values of $\mu$, results which show no evidence of a critical point $[1,2,3]$.

Phenomenological models inspired by the AdS/CFT correspondence $[4,5,6]$ have succeeded in describing some aspects of the quark-gluon plasma [7, 8]. Previous work has mapped the chiral phase transition as a function of temperature and quark chemical potential $[9,10,11]$. In two-flavor models, the phase transition is a crossover for zero quark mass and second order for nonzero quark mass, consistent with lattice QCD models, which find the order of the order of the chiral phase transition to be dependent on the light and strange quark masses. More general arguments also show that the chiral phase transition depends on the number of quark flavors and their masses [12], as summarized in the Columbia plot, which indicates that a first-order transition is achievable in 2+1flavor models. Different holographic models have shown evidence of a critical point by examining baryon susceptibilities [13, 14], but these models do not examine chiral symmetry restoration, which is the focus of this work.

We present the results on the chiral dynamics and meson melting of a flavor-symmetric softwall AdS/QCD model first published in [9]. We extend our analysis to consider the effects of strange quarks on the chiral phase transition. By considering heavy quarks we further our exploration of the QCD phase diagram, specifically the Columbia Plot which characterizes the phase transition as a function of $m_{u, d}$ and $m_{s}$ [15]. We explore a 3-flavor symmetric model ( $m_{l}=m_{u, d}=$ $\left.m_{s}\right)$ and a $2+1$-flavor model $\left(m_{l}=m_{u, d} \neq m_{s}\right)$, extending the work of [16] to finite quark chemical potential.

\section{2-Flavor Model}

To consider the thermodynamics of AdS/QCD, we use an asymptotically anti-de Sitter 5-D black hole metric

$$
d s^{2}=\frac{L^{2}}{z^{2}}\left(-f(z) d t^{2}+d x_{i}^{2}+\frac{d z^{2}}{f(z)}\right) .
$$

Following the procedure of our published work [9] and established in [17, 18, 19], we model finite temperature and chemical potential with a charged black hole described by the 5D AdS-ReissnerNordström metric

$$
f(z)=1-\left(1+Q^{2}\right)\left(\frac{z}{z_{h}}\right)^{4}+Q^{2}\left(\frac{z}{z_{h}}\right)^{6},
$$


where $Q=q z_{h}^{3}$ and $0<Q^{2}<2$. The quark chemical potential and temperature are determined by the charge and horizon position

$$
\begin{aligned}
\mu & =\kappa \frac{Q}{z_{h}}, \\
T & =\frac{1}{\pi z_{h}}\left(1-\frac{Q^{2}}{2}\right) .
\end{aligned}
$$

Note that this is the quark chemical potential, with a value one third of the baryon chemical potential. As in [11] we take $\kappa=1$. Any combination of temperature and chemical potential uniquely determines a combination of $z_{h}$ and $q$.

The matter fields of the theory are described by the following action

$$
\mathscr{S}=\frac{1}{2 k} \int d^{5} x \sqrt{-g} e^{-\Phi(z)} \operatorname{Tr}\left[|D X|^{2}+V_{m}(X)+\frac{1}{2 g_{5}^{2}}\left(F_{A}^{2}+F_{V}^{2}\right)\right] .
$$

where $X$ contains the scalar and pseudoscalar meson fields. The scalar potential is

$$
V_{m}(X)=m_{5}^{2}|X|^{2}+4 v_{4}|X|^{4},
$$

where $m_{5}^{2}=-3$ is the mass of the scalar field and $v_{4}=8$ for good phenomenology. The scalar meson field has a $z$-dependent vacuum expectation value (VEV) that describes the chiral symmetry breaking of the model. In a 2-flavor symmetric model, the VEV becomes

$$
\langle X\rangle=\frac{\chi(z)}{2} I,
$$

where $I$ is the $N_{f} \times N_{f}$ identity matrix.

A quadratic IR dilaton $\Phi(z \rightarrow \infty) \sim z^{2}$ is required for linear confinement [20, 21]. In order to dynamically solve for the chiral field, the dilaton must become negative quadratic in the UV limit [22]. One example of a dilaton profile that produces good meson spectra in the zero-temperature limit is

$$
\Phi(u)=-\mu_{1}^{2} z_{h}^{2} u^{2}+\left(\mu_{0}^{2}+\mu_{1}^{2}\right) z_{h}^{2} u^{2}\left[1-\exp \left(-\mu_{2}^{2} z_{h}^{2} u^{2}\right)\right],
$$

with $\mu_{0}=430 \mathrm{MeV}, \mu_{1}=830 \mathrm{MeV}$, and $\mu_{2}=176 \mathrm{MeV}$. We use this dilaton parameterization for both the two-flavor and 2+1-flavor models.

\subsection{Vector and Axial-Vector Meson Melting}

We present the analysis of the vector and axial-vector mesons as representative cases of the behavior of the meson spectral function[10]. The equation of motion for the vector field is obtained by varying the action

$$
\sqrt{-g} e^{-\Phi} \partial_{v} \partial^{v} V_{\mu}(x, z)+\partial_{z}\left(\sqrt{-g} e^{-\Phi} g^{z z} \partial_{z} V_{\mu}(x, z)\right)=0 .
$$

We perform the Kaluza-Klein decomposition of the Fourier-transformed fields $\tilde{V}_{\mu}(q, z)=\tilde{v}(q, z) \tilde{V}_{\mu}^{0}(q)$ and set momentum $q=0$ to obtain

$$
\tilde{v}^{\prime \prime}-\left(\frac{f(u)-u f^{\prime}(u)+u f(u) \Phi^{\prime}(u)}{u f(u)}\right) \tilde{v}^{\prime}+\left(\frac{\omega^{2} z_{h}^{2}}{f(u)^{2}}\right) \tilde{v}=0,
$$


where $u=z / z_{h}$.

The equation of motion for the axial sector is found similarly. The coupling of the axial field to the scalar VEV $\chi(z)$ results in an additional term that is responsible for the vector-axial mass splitting. The equation of motion for the axial meson reads

$$
\tilde{a}^{\prime \prime}-\left(\frac{f(u)-u f^{\prime}(u)+u f(u) \Phi^{\prime}(u)}{u f(u)}\right) \tilde{a}^{\prime}+\left(\frac{\omega^{2} z_{h}^{2}}{f(u)^{2}}-g_{5}^{2} \frac{\chi(u)^{2}}{u^{2} f(u)}\right) \tilde{a}=0 .
$$

The equations of motion are put into a Schrödinger-like form through a Bogoliubov transformation of the fields. For the purposes of demonstration we choose the axial sector. The equation of motion (2.11) becomes

$$
\partial_{r_{*}}^{2} a+\omega^{2} a=V_{s} a,
$$

with the effective potential

$$
V_{s}=f(z)^{2}\left(\frac{\omega_{a}^{\prime 2}}{4}-\frac{\omega_{a}^{\prime \prime}}{2}\right)+f(z) g_{5}^{2} \frac{\chi^{2}}{z^{2}}
$$

where $\omega_{a} \equiv \Phi(z)+\log (z)$ and the tortoise coordinate $r_{*}$ satisfies $\partial_{r_{*}}=-f(z) \partial_{z}$. Additional details are found in [9].

Near the black hole horizon, the potential in (2.12) vanishes, yielding free-particle in-falling $\left(\psi_{-}\right)$and out-going $\left(\psi_{+}\right)$solutions

$$
\psi_{ \pm}=(1-u)^{ \pm i \frac{\omega z_{h}}{4}}
$$

These wavefunctions can be written as linear combinations of the UV solutions $\left(\psi_{1}, \psi_{2}\right)$ as

$$
\begin{aligned}
& \psi_{-}=A_{-} \psi_{1}+B_{-} \psi_{2} \\
& \psi_{+}=A_{+} \psi_{1}+B_{+} \psi_{2} .
\end{aligned}
$$

Here, $\psi_{1}$ represents the normalizable solution. The spectral function is proportional to $\operatorname{Im}\left(A_{-} / B_{-}\right)$ $[23,24,9]$. The peaks in this function can be visualized as the mass values $\omega$ that allow the normalizable UV solution to be connected to the in-falling solution, which is the correct boundary condition at the horizon.

The results plotted in Figure 1 show the first three states for the vector and axial-vector mesons at a range of temperatures. The peaks become less pronounced at higher temperatures, representing the melting of the bound states, beginning with the excited states. The bound states are seen to melt at a temperature below the chiral phase transition. In addition, the mass splitting between the two meson types stays constant, which is consistent with broken chiral symmetry. Increasing the chemical potential produces a melting process that is qualitatively similar.

\subsection{Chiral Phase Transition}

We solve for $\chi(u)$ for each combination of $T, \mu$ with a numerical shooting method, varying $\sigma$ to determine the value that yields a function $\chi(u)$ that does not diverge at the horizon. These results are shown in Figure 2. The chiral phase transition is second-order in the for $m_{q}=0$, becoming crossover at the physical quark mass. Upon inspection, we find that the chiral transition remains of 


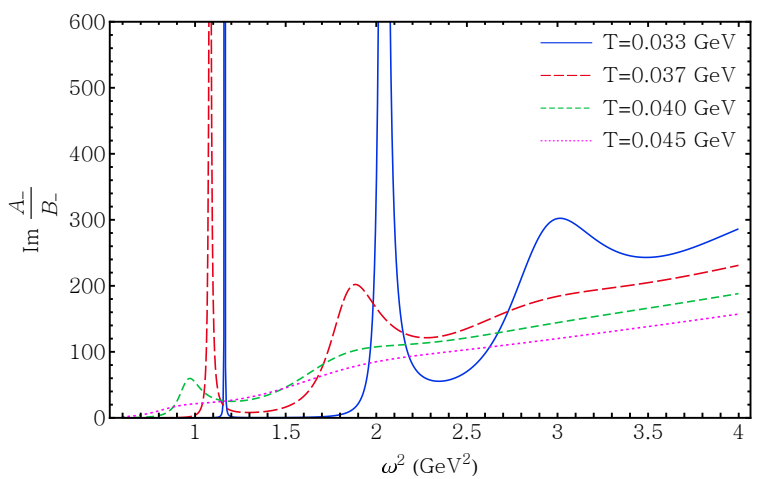

(a)

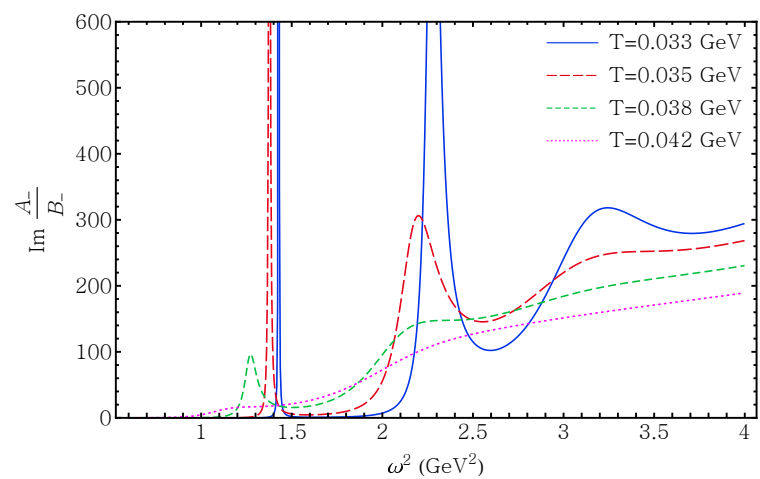

(b)

Figure 1: Spectral functions for (a) vector and (b) axial-vector mesons. The peaks correspond to bound states, which melt at higher temperatures, beginning with the excited states.

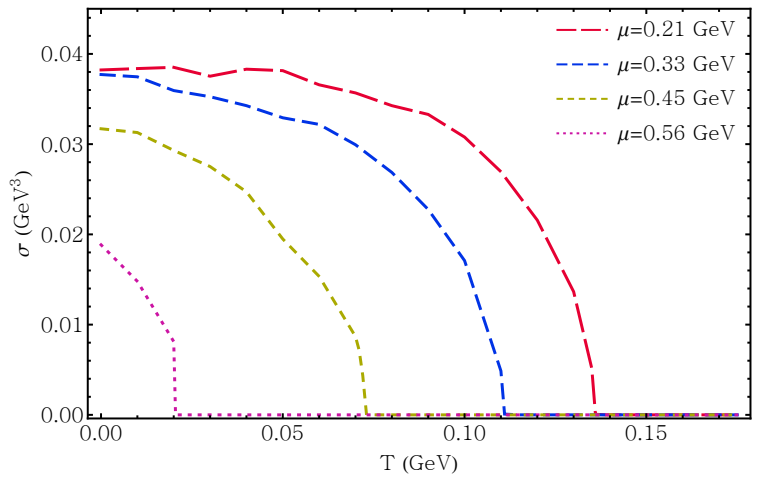

(a)

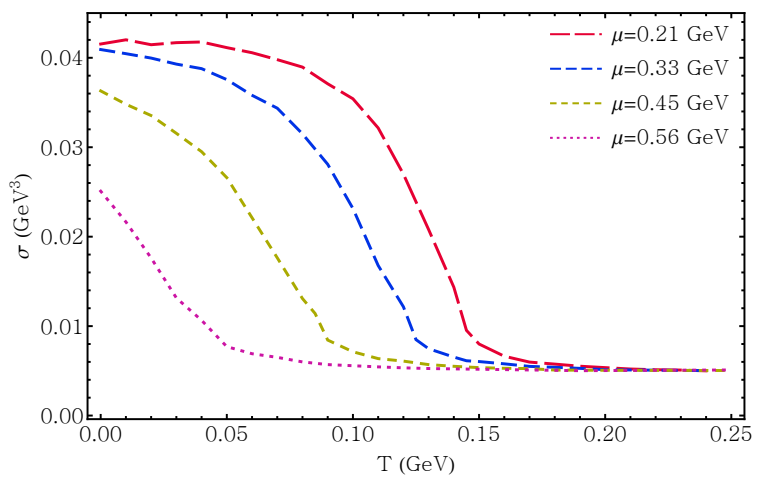

(b)

Figure 2: Dependence of $\sigma$ on temperature for varying $\mu$ values with (a) $m_{q}=0 \mathrm{MeV}$ and (b) $m_{q}=9.75 \mathrm{MeV}$. The transition is second-order in the chiral limit and a crossover for $m_{q} \neq 0$.

the crossover type for small but nonzero quark mass. The effects of $T$ and $\mu$ on chiral symmetry restoration are qualitatively similar.

The quartic term in the scalar potential (2.6) is necessary to achieve the second-order chiral phase transition [20, 25, 26]. Without this term, the sources of spontaneous and explicit chiral symmetry breaking are not independent, causing $\sigma$ to vanish at zero quark mass. Producing a first-order phase transition requires a cubic term in the scalar potential, as investigated in Section 3.

\section{2+1-Flavor Model}

A t'Hooft determinant term in the scalar potential provides mixing between light and heavy flavors [22]. The potential becomes

$$
V_{m}(X)=m_{5}^{2}|X|^{2}+4 v_{4}|X|^{4}+\gamma \operatorname{Re}[\operatorname{det}(X)]
$$




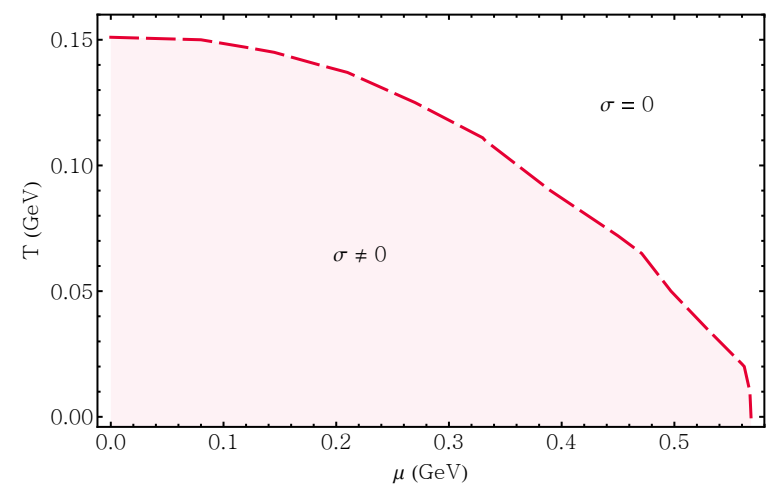

Figure 3: Values of $T$ and $\mu$ for which the quark condensate $\sigma$ goes to zero. The transition is second-order, corresponding to the chiral limit $m_{q}=0$.

where $\gamma=6 \sqrt{2} v_{3}$. As above, we take $v_{4}=8$, and in the following set $v_{3}=-3$. In this $2+1$ flavor model, the scalar VEV takes the form

$$
\langle X\rangle=\frac{1}{\sqrt{2}} \operatorname{diag}\left(\chi_{l}(z), \chi_{l}(z), \chi_{s}(z)\right)
$$

where the normalization factor is chosen to give the kinetic term its canonical form. Thus, the chiral potential becomes

$$
V(\chi)=\left\langle\operatorname{Tr}\left[V_{m}(X)\right]\right\rangle=m_{5}^{2}\left(\chi_{l}^{2}+\frac{1}{2} \chi_{s}^{2}\right)+3 v_{3} \chi_{l}^{2} \chi_{s}+v_{4}\left(2 \chi_{l}^{4}+\chi_{s}^{4}\right) .
$$

Varying 2.5 with respect to $\chi_{l}$ and $\chi_{s}$ respectively yields the following equations of motion,

$$
\begin{gathered}
\chi_{l}^{\prime \prime}-\left(\frac{3 f(u)-u f^{\prime}(u)+u f(u) \Phi^{\prime}(u)}{u f(u)}\right) \chi_{l}^{\prime}+\frac{1}{u^{2} f(u)}\left(3 \chi_{l}-3 v_{3} \chi_{l} \chi_{s}-4 v_{4} \chi_{l}^{3}\right)=0, \\
\chi_{s}^{\prime \prime}-\left(\frac{3 f(u)-u f^{\prime}(u)+u f(u) \Phi^{\prime}(u)}{u f(u)}\right) \chi_{s}^{\prime}+\frac{1}{u^{2} f(u)}\left(3 \chi_{s}-3 v_{3} \chi_{l}^{2}-4 v_{4} \chi_{s}^{3}\right)=0 .
\end{gathered}
$$

The UV behavior of the chiral fields are determined by the AdS/CFT dictionary

$$
\chi_{l}(u \rightarrow 0)=m_{l} \zeta z_{h} u+\frac{\sigma_{l}}{\zeta} z_{h}^{3} u^{3}, \chi_{s}(u \rightarrow 0)=m_{s} \zeta z_{h} u+\frac{\sigma_{s}}{\zeta} z_{h}^{3} u^{3},
$$

where $m_{l}=m_{u, d}$ is the light quark mass, $m_{s}$ is the strange quark mass, and $\sigma_{l}, \sigma_{s}$ are the chiral condensates. In Section 3.1 we analyze the chiral phase transition in the $S U(3)$ case where $m_{l}=m_{s}$, and in Section 3.2 treat the 2+1-flavor case where $m_{l} \neq m_{s}$.

\subsection{Flavor-Symmetric Chiral Phase Transition}

We begin our analysis with the flavor-symmetric case. By setting $m_{l}=m_{s}$ we find $\chi_{l}=\chi_{s}$, so that (3.4) and (3.5) reduce to the single equation of motion

$$
\chi^{\prime \prime}-\left(\frac{3 f(u)-u f^{\prime}(u)+u f(u) \Phi^{\prime}(u)}{u f(u)}\right) \chi^{\prime}+\frac{1}{u^{2} f(u)}\left(3 \chi-3 v_{3} \chi^{2}-4 v_{4} \chi^{3}\right)=0,
$$


subject to the UV boundary condition 3.6 set by the AdS/CFT dictionary. Using a numerical shooting method, we tune the chiral condensate $\sigma$, finding solutions to (3.7) that are normal at the black hole horizon. At values of $T, \mu$ where such solutions do not exist, the trivial solution $\chi=0$ is realized, signifying chiral symmetry restoration. As pointed out in [22], the inclusion of the cubic t'Hooft determinant term in the scalar potential breaks the $\chi \leftrightarrow-\chi$ symmetry, giving energetic preference to positive chiral fields. As a result, the $\sigma \leftrightarrow-\sigma$ symmetry found in the two-flavor case is also broken, allowing first-order phase transitions.

For a given quark mass, we solve for the chiral condensate as a function of temperature and baryon density. Figure 4 shows examples of first-order, second-order, and crossover transitions for representative values of the quark mass and chemical potential. The critical value of the quark mass separating first-order and crossover phase transitions is $m_{q}=35 \mathrm{MeV}$. This critical quark mass is found to be independent of $\mu$. This implies that there is no critical point in the $T-\mu$ plane in this flavor-symmetric model.

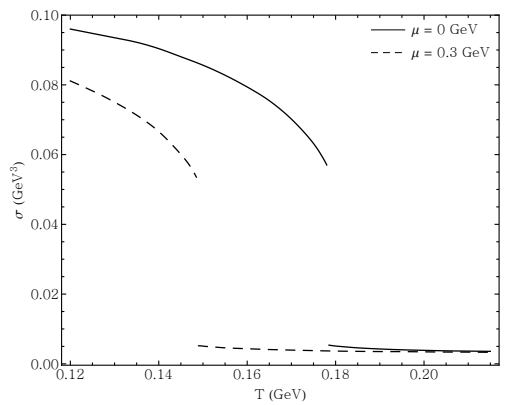

(a)

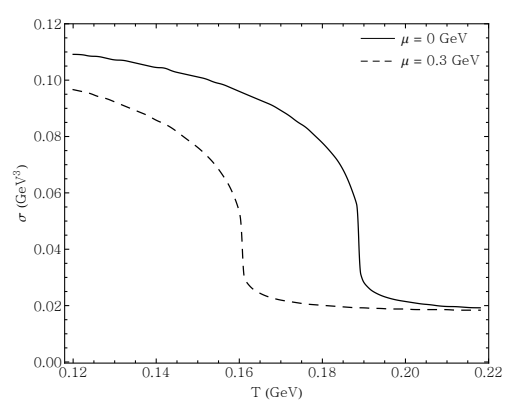

(b)

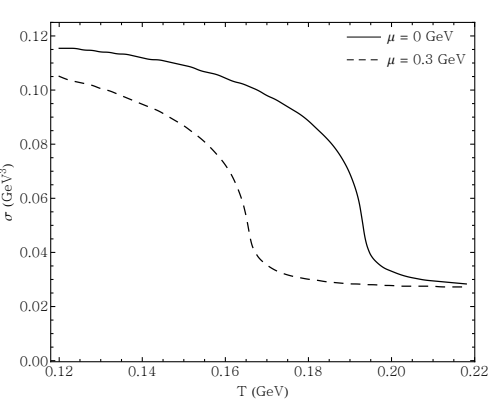

(c)

Figure 4: Dependence of $\sigma$ on $T$, for zero and non-zero values of $\mu$ and (a) $m_{l}=m_{s}=10 \mathrm{MeV}$, (b) $m_{l}=m_{s}=35 \mathrm{MeV}$, and (c) $m_{l}=m_{s}=45 \mathrm{MeV}$. In all cases, chemical potential has no effect on the order of the phase transition.

\subsection{2+1-Flavor Chiral Phase Transition}

Finding no critical point in the flavor-symmetric case, we extend the model to $2+1$ flavors. We use the shooting method to solve $(3.4,3.5)$, searching for values of $\sigma_{l}$ and $\sigma_{s}$ that yield regular solutions for both chiral fields. A representative case is shown for $m_{l}=40 \mathrm{MeV}, m_{s}=70 \mathrm{MeV}$. From Figure 5 it is clear that $\sigma_{l}$ and $\sigma_{s}$ do not coincide. In fact, by examining the case where $m_{l}=0 \mathrm{MeV}, m_{s}=200 \mathrm{MeV}$, we find that $\sigma_{l}$ undergoes a second-order transition at all values of $\mu$ whereas the phase transition for $\sigma_{s}$ is first-order. Thus we find that the order of the transition is not necessarily the same for the two flavors, though no combination of quark masses produces a first-order transition for one flavor and crossover for the other. In the case that the orders do not agree, we characterize the transition as second-order.

As a summary of our analysis, we plot the phase structure of the mass plane for $\mu=0 \mathrm{MeV}$ and $\mu=300 \mathrm{MeV}$. As shown in Figure 6, when $m_{s}$ is small we identify slight discrepancies on the order of $1 \mathrm{MeV}$ which we deem insignificant. We find that the order of a transition is unchanged at finite chemical potential. 


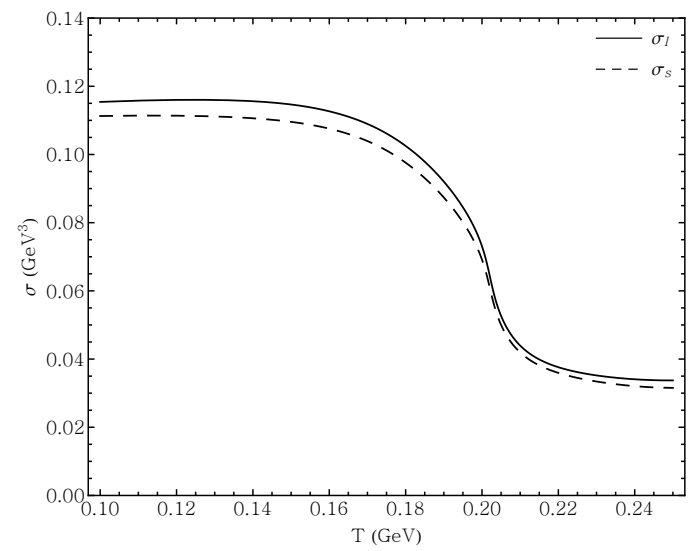

(a)

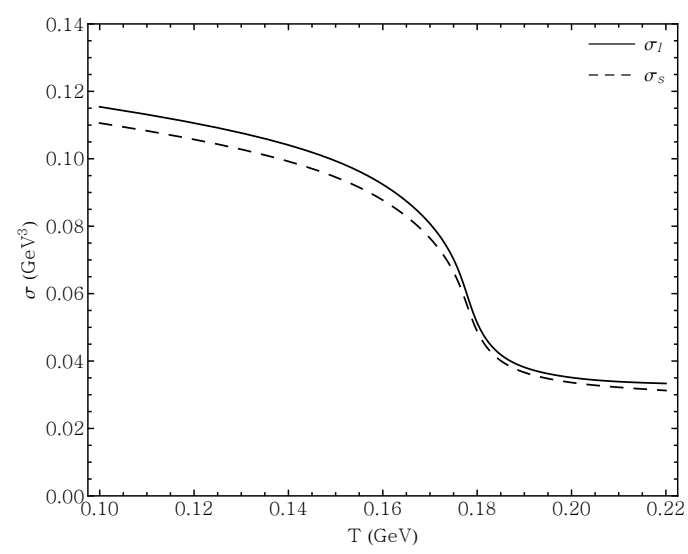

(b)

Figure 5: Dependence of $\sigma_{l}$ and $\sigma_{s}$ on $T$ with $m_{l}=40 \mathrm{MeV}, m_{s}=70 \mathrm{MeV}$ and (a) $\mu=0 \mathrm{MeV}$ and (b) $\mu=300 \mathrm{MeV}$.

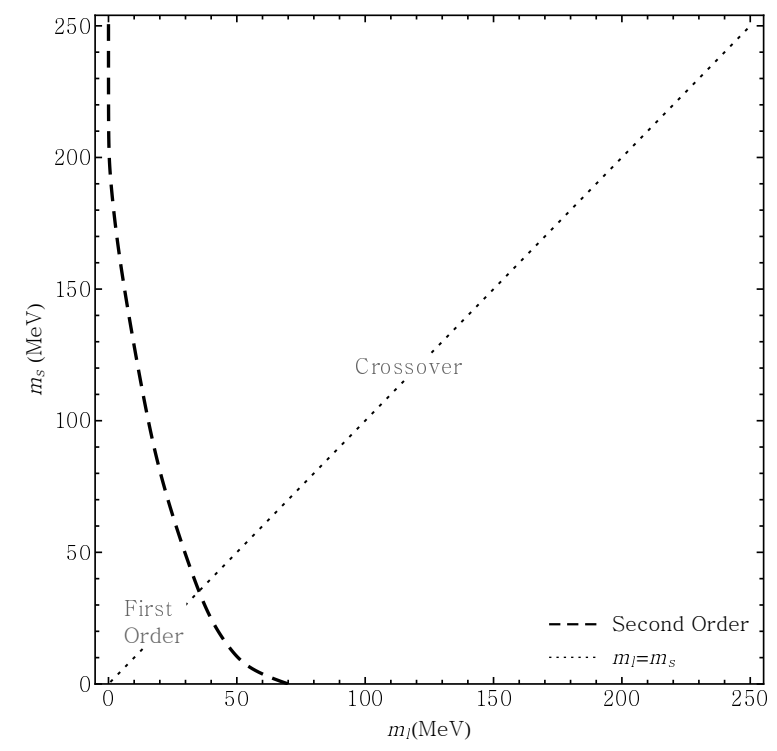

(a)

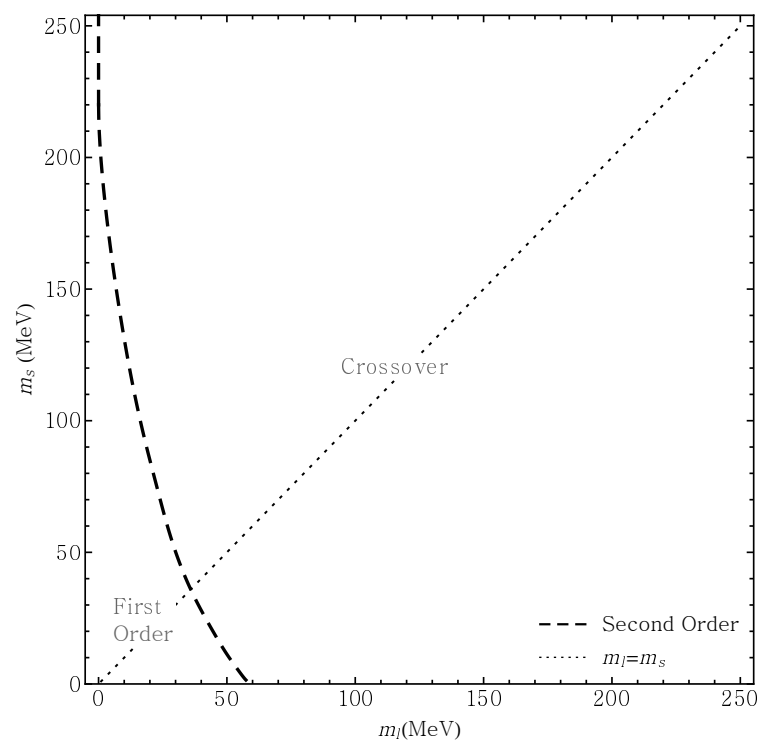

(b)

Figure 6: The Columbia Plot produced by the $2+1$-flavor model for (a) $\mu=0 \mathrm{MeV}$ and (b) $\mu=300$ $\mathrm{MeV}$.

\section{Conclusion and Discussion}

In this work, we investigate the chiral phase transition in soft-wall AdS/QCD at finite temperature and quark chemical potential. The scalar VEV is modified to include strange quarks, and higher order terms in the scalar potential are including in the action. A quartic term allows for independent sources of explicit and spontaneous chiral symmetry breaking, while a cubic t'Hooft determinant term allows for flavor mixing and first-order phase transitions. All analysis is performed in the finite chemical potential regime, using the AdS-Reissner-Nordström metric. Using 
the shooting method we numerically solve for the chiral field and extract the dependence of the chiral condensates on temperature and baryon density.

We first present the degenerate case where $m_{q}=m_{u / d}=m_{s}$, and find that the value $m_{q}=35$ $\mathrm{MeV}$ separates first-order from crossover transitions. In agreement with lattice results and other nonperturbative methods, we find the second-order curve that separates first-order from crossover transitions by allowing $m_{s} \neq m_{l}$. When $m_{s}$ is large, our results are consistent with the two flavor model, where the transition is second-order in the chiral limit.

This paper presents improvements upon earlier results in this area by incorporating finite chemical potential to a three flavor model, enabling rich exploration of chiral dynamics. This holographic model admits no critical point in the $T-\mu$ plane. Finite baryon number density has no effect on the order of the transition, regardless of quark mass. In particular this model couples all quarks to the quark chemical potential, and is qualitatively symmetric between $T$ and $\mu$. Future work in this area should provide qualitative differences at finite quark chemical potential. One possibility is to couple quark chemical potential to the light quarks only. In addition, the dilaton and metric should be solved dynamically from the gravity action, rather than being parameterized by hand.

\section{References}

[1] P. de Forcrand and O. Philipsen, The Chiral critical line of $N(f)=2+1$ QCD at zero and non-zero baryon density, JHEP 01 (2007) 077, [hep-lat/ 0607017 ].

[2] C. Bonati, P. de Forcrand, M. D'Elia, O. Philipsen and F. Sanfilippo, The chiral phase transition in two-flavor QCD from imaginary chemical potential, Physical Review D 90 (aug, 2014) 074030, [1408.5086].

[3] R. Bellwied, S. Borsányi, Z. Fodor, J. Günther, S. Katz, A. Pásztor et al., Towards the QCD phase diagram from analytical continuation, Nuclear Physics A 956 (dec, 2016) 797-800, [1601.00466].

[4] J. Maldacena, The large $N$ limit of superconformal field theories and supergravity, Advances in Theoretical and Mathematical Physics 2 (1998) 231-252, [hep-th/9711200].

[5] E. Witten, Anti-de Sitter space, thermal phase transition, and confinement in gauge theories, Advances in Theoretical and Mathematical Physics 2 (1998) 505-532, [hep-th/9803131].

[6] A. Karch, E. Katz, D. Son and M. Stephanov, Linear confinement and AdS/QCD, Physical Review D $74(7,2006)$ 015005, [hep-ph / 0602229$].$

[7] A. Vega and A. Ibañez, Analysis of soft wall AdS / QCD potentials to obtain melting temperature of scalar hadrons, 1706.01994.

[8] R. Zöllner and B. Kämpfer, Extended soft wall model with background related to features of QCD thermodynamics, The European Physical Journal A 53 (jun, 2017) 139, [1701.01398].

[9] S. P. Bartz and T. Jacobson, Chiral Phase Transition and Meson Melting from AdS/QCD, Phys. Rev. D94 (2016) 075022, [1607.05751].

[10] L.-X. Cui and Y.-L. Wu, Thermal Mass Spectra of Scalar and Pseudo-Scalar Mesons in IR-improved Soft-Wall AdS/QCD Model with Finite Chemical Potential, Modern Physics Letters A 28 (2013) 14, [1302.4828v2]. 
[11] P. Colangelo, F. Giannuzzi, S. Nicotri and V. Tangorra, Temperature and quark density effects on the chiral condensate: an AdS/QCD study, The European Physical Journal C, Volume 72, Issue 8, 2096 (2012) 72 (2012), [1112.4402].

[12] R. D. Pisarski and F. Wilczek, Remarks on the chiral phase transition in chromodynamics, Phys. Rev. D 29 (Jan, 1984) 338-341.

[13] R. Critelli, J. Noronha, J. Noronha-Hostler, I. Portillo, C. Ratti and R. Rougemont, Critical point in the phase diagram of primordial quark-gluon matter from black hole physics, 1706.00455.

[14] Z. Li, Y. Chen, D. Li and M. Huang, Locating the QCD critical end point through the peaked baryon number susceptibilities along the freeze-out line, 1706.02238.

[15] E. Laermann and O. Philipsen, The Status of lattice QCD at finite temperature, Ann. Rev. Nucl. Part. Sci. 53 (2003) 163-198, [hep-ph/0303042].

[16] D. Li and M. Huang, Chiral phase transition of qcd with $n f=2+1$ flavors from holography, Journal of High Energy Physics 2017 (2017) 42.

[17] A. Chamblin, R. Emparan, C. V. Johnson and R. C. Myers, Charged AdS black holes and catastrophic holography, Physical Review D 60 (8, 1999) 064018, [hep-th/9902170].

[18] C. Park, Dissociation of a heavy meson in the quark medium, Physical Review D 81 (2, 2010) 045009, [0907.0064].

[19] P. Colangelo, F. Giannuzzi and S. Nicotri, Holography, heavy-quark free energy, and the QCD phase diagram, Physical Review D 83 (2, 2011) 035015, [1008.3116].

[20] A. Karch, E. Katz, D. T. Son and M. A. Stephanov, Linear confinement and AdS/QCD, Physical Review D 74 (7, 2006) 015005, [hep-ph/ 0602229 ].

[21] A. Karch, E. Katz, D. T. Son and M. A. Stephanov, On the sign of the dilaton in the soft wall models, Journal of High Energy Physics 2011 (4, 2011) 66, [1012. 4813].

[22] K. Chelabi, Z. Fang, M. Huang, D. Li and Y.-L. Wu, Chiral phase transition in the soft-wall model of AdS/QCD, Journal of High Energy Physics 2016 (4, 2016) 36, [1512 . 06493 ].

[23] A. S. Miranda, C. Ballon Bayona, H. Boschi-Filho and N. R. Braga, Glueballs at finite temperature from AdS/QCD, Nuclear Physics B - Proceedings Supplements 199 (2, 2010) 107-112, [0910.4319].

[24] A. S. Miranda, C. Ballon Bayona, H. Boschi-Filho and N. R. Braga, Black-hole quasinormal modes and scalar glueballs in a finite-temperature AdS/QCD model, Journal of High Energy Physics 2009 $(11,2009)$ 119-119, [0909.1790].

[25] T. Gherghetta, J. Kapusta and T. Kelley, Chiral symmetry breaking in the soft-wall AdS/QCD model, Physical Review D 79 (4, 2009) 076003, [hep-ph/0902.1998].

[26] S. Bartz and J. Kapusta, Dynamical three-field AdS/QCD model, Physical Review D 90 (2014), [1406.3859]. 